\title{
AVALIAÇÃO PÓS-OCUPAÇÃO DAS CONDIÇÕES DE HABITAÇÃO EM CONJUNTOS HABITACIONAIS DO PMCMV EM BLUMENAU SC
}

\author{
B. C. HORONGOSO, A. J. BOGO \\ Universidade Regional de Blumenau - FURB \\ bogo.amilcar@gmail.com
}

Submetido 16/04/2018 - Aceito 05/11/2018

DOI: 10.15628/holos.2018.7210

\section{RESUMO}

Neste trabalho é apresentada uma análise de avaliação pós-ocupação - APO das condições de habitação em três (3) conjuntos habitacionais edificados pelo Programa Minha Casa Minha Vida - PMCMV em Blumenau SC, edificações estas destinadas para famílias desalojadas de suas casas devido às tragédias ambientais (enxurradas, alagamentos, deslizamentos e enchentes) ocorridas em 2008 no município. Por meio de análises de projeto, visitas in loco, vistorias técnicas, aplicação de questionários e entrevistas foram realizadas análises englobando avaliação de conforto ambiental, funcionalidade, ambiência urbana e sustentabilidade. A partir da análise geral foram selecionados três Conjuntos Habitacionais para análises mais detalhadas de avaliação como antes citado, no caso os nominados como conjuntos Hamburgo, Morada das Figueiras e Parque da Lagoa, localizados na região oeste do município. De forma geral os resultados apontam que existem nos três (3) Conjuntos Habitacionais avaliados uma padronização da tipologia dos apartamentos, não considerando a diversidade dos núcleos familiares; falta de estratégias arquitetônicas quanto ao conforto ambiental nos apartamentos e inserção urbana ineficiente, destacando-se a baixa oferta de transporte coletivo, espaços de lazer, comércio e serviços de apoio. Também foi identificado ainda um sentimento de abandono por parte dos moradores e facilidade da entrada do tráfico de drogas nos espaços dos condomínios gerando insegurança.

PALAVRAS-CHAVE: Avaliação Pós-ocupação, Sustentabilidade, Conforto ambiental, Conjuntos habitacionais, PMCMV.

\section{POST-OCCUPANCY EVALUATION (APO) OF HOUSING CONDITIONS IN HOUSING ESTATES BUILT BY PMCMV PROGRAM - IN BLUMENAU SC}

\begin{abstract}
This paper presents a post-occupancy evaluation (APO) analysis of housing conditions in three (3) housing estates built by the Minha Casa Minha Vida Program - PMCMV in Blumenau SC, designed for homeless families from their homes because of environmental disasters (floods, waterlogging and floods) that have occurred in 2008 in the city. Through design reviews, site visits, technical surveys, questionnaires, interviews, analyzes were carried out encompassing evaluation of environmental comfort, functionality, urban ambience and sustainability. From this first analysis we selected three inhabitation areas for more detailed analysis of evaluation as mentioned before, if the nominees as the Hamburgo, Morada das Figueiras and Parque da Lagoa, located in the western region of the county. Overall the results show that there are the three (3) housing estates evaluated a standardized typology of apartments, not considering the diversity of family units; lack of architectural strategies regarding environmental comfort in the apartments and inefficient urban integration, highlighting the low public transport supply, leisure facilities, trade and support services. Also identified was still a feeling of abandonment by the residents and ease of drug trafficking in entry spaces condominiums generating insecurity.
\end{abstract}

KEYWORDS: Post-occupancy evaluation; Sustainability; Environmental comfort; Housing estates; PMCMV. 


\section{INTRODUÇÃO}

A utilização de técnicas de avaliação pós-ocupação APO no Brasil vêm crescendo continuamente no meio acadêmico, destacando-se trabalhos de APO principalmente para edifícios escolares, conjuntos habitacionais, edifícios de escritórios, hospitais, edifícios universitários, entre outros. Estes trabalhos avaliaram edificações e espaços construídos a partir do ponto de vista do grau de satisfação do usuário, permitindo uma avaliação crítica quanto à qualidade da arquitetura produzida em relação às necessidades do ser humano.

O trabalho desenvolvido realizou uma análise de avaliação pós-ocupação - APO das condições de habitação em edificações residenciais de habitação de interesse social do Programa Minha Casa Minha Vida no município de Blumenau SC, localizado na região do médio vale do Itajaí (a cerca de $60 \mathrm{~km}$ do litoral norte de Santa Catarina), onde vivem aproximadamente $344 \mathrm{mil}$ habitantes (IBGE, 2016).

Foi realizado o levantamento de informações quanto as relações ser humano $\mathrm{x}$ ambiente construído em edificações residenciais, numa amostra de três (3) conjuntos habitacionais da cidade.

O estudo possibilitou uma avaliação crítica da situação existente e a definição de alternativas para a melhoria da qualidade de vida junto aos moradores das edificações residenciais.

\section{REVISÃO BIBLIOGRÁFICA}

Segundo Ornstein e Romero (1992), a metodologia de avaliação pós-ocupação APO, pretende a partir da avaliação de fatores técnicos, funcionais, econômicos, estéticos e comportamentais do ambiente em uso, e tendo em vista a opinião dos técnicos, projetistas e clientes, como também dos usuários, diagnosticar aspectos positivos e negativos, definindo para este último caso, recomendações que minimizem ou corrijam os problemas detectados e realimentem o ciclo de produção de projetos arquitetônicos semelhantes.

Em relação a avaliação em si, o mesmo autor afirma que qualquer ambiente construído ou conjunto de ambientes construídos independentemente da complexidade e escala, é passível de avaliação.

Muitas das avaliações pós-ocupação são desenvolvidas sob enfoques específicos, como de conforto ambiental, patologias construtivas, comportamentais, entre outras, com vistas a aferição do nível de satisfação dos usuários, seja em partes ou no todo. Neste sentido, diversos trabalhos de avaliação pós-ocupação APO em edificações foram realizados no Brasil, para cidades diversas em diferentes regiões e estados, como São Paulo, Rio de Janeiro, Florianópolis, Porto Alegre, Brasília, Cuiabá, São Carlos-SP, Natal, Recife, Blumenau, entre outras.

Sobre avaliação pós-ocupação, Kowaltowski et al (2009) apresentam os conceitos de satisfação e valor desejado neste tipo de avaliação pós-ocupação em habitação social para um 
estudo de caso na região de Campinas, SP, avaliando a percepção do morador de conjuntos habitacionais.

Já Coelho-de-Souza et al (2009), avaliaram a percepção de conforto ambiental na habitação popular Casa Alvorada, em uma terceira avaliação pós-ocupação em um conjunto de habitações idealizadas pelo NORIE/UFRGS e construídas pela prefeitura de Nova Hartz/RS, identificando a necessidade de avaliações contínuas e rotineiras.

Também focado no conforto ambiental como objetivo de uma avaliação pós-ocupação, Mendes e Silveira (2010) avaliaram um conjunto habitacional multifamiliar em Teresina PI, identificando pontos positivos e negativos às habitações avaliadas.

Vergara et al (2007), realizaram uma avaliação pós-ocupação (APO) em ambientes residenciais localizados na cidade de Santa Maria RS, cuja ênfase é dada aos aspectos ergonômicos aplicados ao ambiente construído, a partir da análise da composição dos ambientes residenciais definidos pelas funções estar, serviço e dormir, ou seja, aqui o foco da APO está nestas funções.

Do ponto de vista metodológico em avaliação pós-ocupação, Rodrigues et al (2006), apresentam ferramenta adaptada de uma matriz de descobertas e recomendações. Esta ferramenta foi utilizada em uma avaliação das edificações hospitalares do Instituto Fernandes Figueira (IFF) no Rio de Janeiro RJ, com vistas à elaboração de projeto de requalificação e redistribuição espacial, identificando os principais problemas e patologias do conjunto de edifícios.

Outro trabalho sobre avaliação pós-ocupação é o de Carmo Filho e Veloso (2005), que realizaram a avaliação de quatro residências em Natal RN, cujos projetos procuraram atender às recomendações do livro Roteiro para construir no Nordeste: Arquitetura como lugar ameno nos trópicos ensolarados de Armando Holanda, considerada uma obra literária pioneira no Brasil acerca do conforto no projeto de arquitetura.

Este último trabalho apresenta uma ideia comum de muitos trabalhos de avaliação pósocupação: identificação de erros e acertos de projeto, visando realimentação do processo de concepção arquitetônica.

A avaliação pós-ocupação - APO permite levantar diferentes aspectos de qualidade, tanto de conforto ambiental como de outras áreas, a partir principalmente do ponto de vista dos usuários, complementando através de analises e inspeções técnicas de campo, juntamente com outras analises abrangidas em outros métodos de avaliação de edificações mais restritos.

Uma das vantagens deste tipo de método, é que permite a avaliação de diferentes aspectos de uma só vez sob a perspectiva dos usuários, ou seja, quem utiliza a edificação, que conhece o cotidiano da sua utilização, expostas às reais condições de uso.

No enfoque de edifícios ofertados pelo mercado imobiliário para as classes de renda média, pode-se citar Villa, Saramago e Alves (2015), que diagnosticaram a qualidade ambiental de empreendimentos construídos em duas cidades de porte médio no Brasil (Uberlândia/MG e Ribeirão Preto/SP). A avaliação de qualidade ambiental nos edifícios analisados foi realizada com base nas estratégias de sustentabilidade citadas em diferentes métodos de certificação de 
edificações, (AQUA, LEED, Casa Azul CAIXA), identificando nos mesmos as situações existentes, como as mais recorrentes, quanto aquelas com maior valor de pontuação dos métodos. Os autores apontam para a incipiente incorporação de estratégias de sustentabilidade nos edifícios investigados, indicando um baixo nível de consciência ambiental dos moradores.

Ornstein, Ono e Oliveira (2017) realizaram trabalho sobre instrumentos para a Avaliação Pós-Ocupação (APO) com vistas à avaliação de empreendimentos habitacionais de interesse social e construído com sistemas denominados "inovadores". Estes autores citam que " $O$ aperfeiçoamento dos formulários e dos procedimentos de aplicação dos instrumentos $e$ da realização das medições e, especialmente, a aplicação de uma APO completa, permitiu observar a relevância da avaliação de desempenho em uso no caso de sistemas construtivos chamados inovadores e adotados em diversas regiões geográficas e climáticas do país. Ficou evidente que não basta a realização da avaliação de desempenho do sistema construtivo inovador, pois muitas vezes esse sistema construtivo é apenas um dos componentes da unidade habitacional. Ao morador / usuário final não importa o desempenho do sistema construtivo inovador isolado, mas do conjunto da edificação e seu potencial de ampliação, que só é possível avaliar, após a obra pronta e ouvindo também o usuário".

Ornstein (2017) apresenta uma reflexão crítica sobre o estágio atual das pesquisas em Avaliação Pós-Ocupação (APO) no Brasil, identificando que após trinta anos, existem poucas teorias e avanços nas abordagens metodológicas. Ao mesmo tempo cita que "nos últimos 20 anos a APO sofreu diversos aprofundamentos em suas abordagens metodológicas, o que vem significando avanços acadêmicos gradativos, mesmo que em alguns casos os resultados ainda sejam tímidos e careçam de maior desenvolvimento".

Sobre a construção de Conjuntos Habitacionais do Programa PMCMV no Brasil, Amore (2015) cita situações em que "O padrão de inserção urbana, promove "frentes pioneiras" que são constituídas a partir de implantação de novos conjuntos em franjas periurbanas descontínuas, muitas vezes fora do perímetro urbano, já existente como expandem os empreendimentos do programa para territórios com problemas ambientais e perto de setores vulneráveis socialmente. $O$ acesso a muitos desses empreendimentos é feito por via única ou rodovia. Em muitas situações os pontos de captação de água e tratamento de esgoto são feitos por estações de esgoto compactas, gerando problemas na execução e manutenção devido a baixa infraestrutura das áreas as quais os empreendimentos são implementados".

Villa et al (2016) identifica que autores clássicos da literatura sobre APO atestam a relevância deste método de avaliação para o conhecimento dos resultados nas decisões de projeto. Estes autores tratam "do aprimoramento de procedimentos metodológicos de APO funcional, comportamental e ambiental em edifícios de apartamentos através do desenho e utilização de interfaces digitais especificamente desenvolvidas". Os autores trabalharam no desenvolvimento de interfaces digitais para coleta e tratamento de dados, como uso de softwares, dispositivos tipo tablet, banco de dados em ambiente web, facilitando a coleta, tratamento e análise de resultados de uma APO.

A utilização da avaliação pós-ocupação - APO como uma metodologia de avaliação crítica de edifícios residenciais de habitação de interesse social em Blumenau, caracteriza-se como 
consolidada do ponto de vista conceitual, permitindo uma análise fundamentada sobre a produção arquitetônica local.

Sobre APO e as unidades habitacionais construídas no Programa Minha Casa Minha Vida PMCMV em Blumenau, Palermo e Peixer (2013) realizaram estudo sobre o perfil populacional de dois conjuntos habitacionais e o rebatimento no espaço da moradia.

As mesmas autoras informam que este programa iniciou cerca de 02 anos após os desastres naturais de 2008 (que geraram grande aumento na demanda por habitação de interesse social na cidade), com a conclusão de 1824 unidade habitacionais (UH) distribuídas em 7 conjuntos (residenciais) na cidade, de 96 até $580 \mathrm{UH}$, com apartamentos de 02 dormitórios em $42 \mathrm{~m}^{2}$ de área privativa.

\section{METODOLOGIA}

A análise de avaliação pós-ocupação - APO das condições de habitação em três (3) conjuntos habitacionais edificados pelo Programa Minha Casa Minha Vida - PMCMV em Blumenau SC foi realizada com base nos seguintes procedimentos metodológicos:

- Caracterização geral dos conjuntos habitacionais do PMCMV em Blumenau e seleção da amostra de estudo;

- Levantamento de dados de campo e de escritório: mapas, plantas, especificações de projeto, registro fotográfico;

- Análises quanto às condições de habitação: avaliação de conforto ambiental, funcionalidade, ambiência urbana e sustentabilidade, a partir de análises de projeto, visitas in loco, vistorias técnicas, aplicação de questionários, entrevistas.

Devido à grande dificuldade de encontrar moradores dispostos a colaborar com as questões de análise, seja por não estarem presentes durante as abordagens de campo, inibição, outros motivos, toda resposta recebida nos questionários e entrevistas foi aproveitada, uma vez que o percentual de respondentes foi muito baixo (de 04 a 18, dependendo do conjunto analisado). Assim, figuras-chave no cotidiano dos conjuntos habitacionais pesquisados foram de suma importância, no caso aqueles com alguma função em cada bloco edificado (subsíndico) ou no total (zeladores). As pesquisas de campo foram realizadas em datas diferentes, durante o primeiro semestre de 2016.

\subsection{Análises Realizadas}

As análises realizadas se basearam na identificação da avaliação da situação de adequação total (Adequado), parcial (Parcial) e Inadequação (Inadequado), referente a:

Conforto ambiental: avaliação quanto controle solar nas aberturas \& luz natural controlada; iluminação natural nos BWCs; ventilação natural cruzada; tratamento paisagístico com sombra arbórea no térreo; Desempenho térmico de coberturas e paredes externas;

Funcionalidade: avaliação quanto à dimensão dos ambientes;

Ambiência urbana: avaliação quanto a existência de: 
Equipamentos de apoio como: escola, creche, posto de saúde ou ambulatório, comércio/serviços, praças/parques/equipamentos de lazer, transporte público;

Áreas de convivência, no caso referente a:

Concentração e tratamento de espaços abertos:

$\rightarrow$ diferenciação entre espaços abertos e estacionamentos, definindo funcionalmente os espaços;

$\rightarrow$ demarcação de limites de segurança; privacidade e identidade e o conforto no uso destes lugares;

Segregação e tratamento de acessos:

$\rightarrow$ acessibilidade e segurança dos acessos desde a rua;

$\rightarrow$ diferenciação dos percursos destinados aos pedestres e ao trânsito de

veículos;

Características das vias circundantes aos edifícios:

$\rightarrow$ necessidade de segurança e boa ambiência, apresentando conforto acústico e higiene;

Racionalização da implantação dos estacionamentos:

$\rightarrow$ proporcionar redução do fluxo veicular, da área pavimentada e sem

ocupar áreas verdes;

Localização das lixeiras coletivas:

$\rightarrow$ necessidade de salubridade, em lugar fechado e longe de espaços de uso

público;

Cobertura na entrada dos edifícios:

$\rightarrow$ necessidade de proteção contra intempéries, proporcionando demarcação de acesso principal;

Adequação ao terreno natural:

$\rightarrow$ implantação da edificação com aproveitamento da topografia natural, desníveis e cobertura do terreno;

$\rightarrow$ nível de implantação dos apartamentos térreos para a necessidade de privacidade e ambiência dos cômodos;

Forma e tratamento externo dos edifícios:

$\rightarrow$ definição da morfologia do edifício para aproveitar os fatores climáticos favoráveis, como o vento e a vegetação;

$\rightarrow$ criação favorável ou não de microclimas; necessidade de orientação e identidade.

Sustentabilidade: avaliação quanto a:

Sustentabilidade ambiental, englobando:

$\rightarrow$ Proteção ecológica e agricultura urbana;

$\rightarrow$ Infraestrutura verde: gestão da água, drenagem natural, tratamento de esgoto alternativo;

$\rightarrow$ Redução, reutilização e reciclagem de resíduos;

Sustentabilidade social, englobando:

$\rightarrow$ Urbanidade;

$\rightarrow$ Comunidade com sentido de vizinhança;

$\rightarrow$ Mobilidade e transporte sustentável 


\section{RESULTADOS E DISCUSSÕES}

Na Tabela 1 adiante é apresentada uma caracterização geral dos 3 Conjuntos Habitacionais, identificando informações sobre cada empreendimento, desde a situação geral (localização, tamanho, etc), até das unidades residenciais (área privativa, área útil).

I) Residencial Morada das Figueiras, na Rua Wilson Bornhofen, Passo Manso: 200 apartamentos em 10 blocos de 5 pavimentos cada e 200 vagas de garagem;

II) Residencial Hamburgo, na Rua Heinrich Hemmer, 1583, Badenfurt: 160 apartamentos em 10 blocos de 4 pavimentos cada e 160 vagas de garagem;

III) Residencial Parque da Lagoa, nao Acesso lateral rua Frederico Jensen, Itoupavazinha: 96 apartamentos em 3 blocos de 4 pavimentos cada e 96 vagas de garagem;

Tabela 1: Caracterização dos Conjuntos Habitacionais com suas respectivas áreas em $\mathrm{m}^{2}$.

\begin{tabular}{|c|c|c|c|c|c|}
\hline Conjunto & $\begin{array}{c}\text { Área útil } \\
\text { do apto }\end{array}$ & $\begin{array}{c}\text { Área } \\
\text { privativa do } \\
\text { apto }\end{array}$ & $\begin{array}{c}\text { Área Total } \\
\text { Construída }\end{array}$ & $\begin{array}{c}\text { Área } \\
\text { Total } \\
\text { terreno }\end{array}$ & $\begin{array}{c}\text { Área verde } \\
\text { (permeável) }\end{array}$ \\
\hline I & 37,35 & 41,36 & $9.134,57$ & $11.158,72$ & $\begin{array}{c}3.977,02 \\
(35,64 \%)\end{array}$ \\
\hline II & 38,01 & 44,15 & $7.855,93$ & $18.740,21$ & $\begin{array}{c}10.072,10 \\
(53 \%)\end{array}$ \\
\hline III & 36,29 & 42,06 & $4.437,00$ & $6.267,83$ & $\begin{array}{c}1.080,02 \\
(17,23 \%)\end{array}$ \\
\hline
\end{tabular}

Foram analisados três (3) Conjuntos Habitacionais localizados nos bairros Passo Manso, Badenfurt e Itoupavazinha, que possuem características semelhantes no que diz respeito a sua morfologia, ocupação e desenvolvimento. São bairros em expansão urbana, junto com zonas industriais e terrenos com características rurais. Não possuem morros altos, sendo um ponto positivo para ocupação urbana, entretanto ainda cobertos por densa vegetação.

Os três bairros possuem grandes vazios urbanos, pouco comércio e serviços de apoio aos moradores, com baixa vitalidade urbana e sentimento de bairro, estimulando maiores deslocamentos até regiões centrais. Os estabelecimentos nesses bairros são, geralmente, voltados aos transportes de cargas e automóveis em geral, devido ao elevado trânsito de veículos que se encontram nas ruas principais desses bairros. O prejuízo maior desses fatos acontece no bairro Badenfurt, ao qual é cortado ao meio pela BR 470, segregando o bairro.

Os três Conjuntos habitacionais são adiantes localizados nos bairros descritos na Figura 1 adiante: 


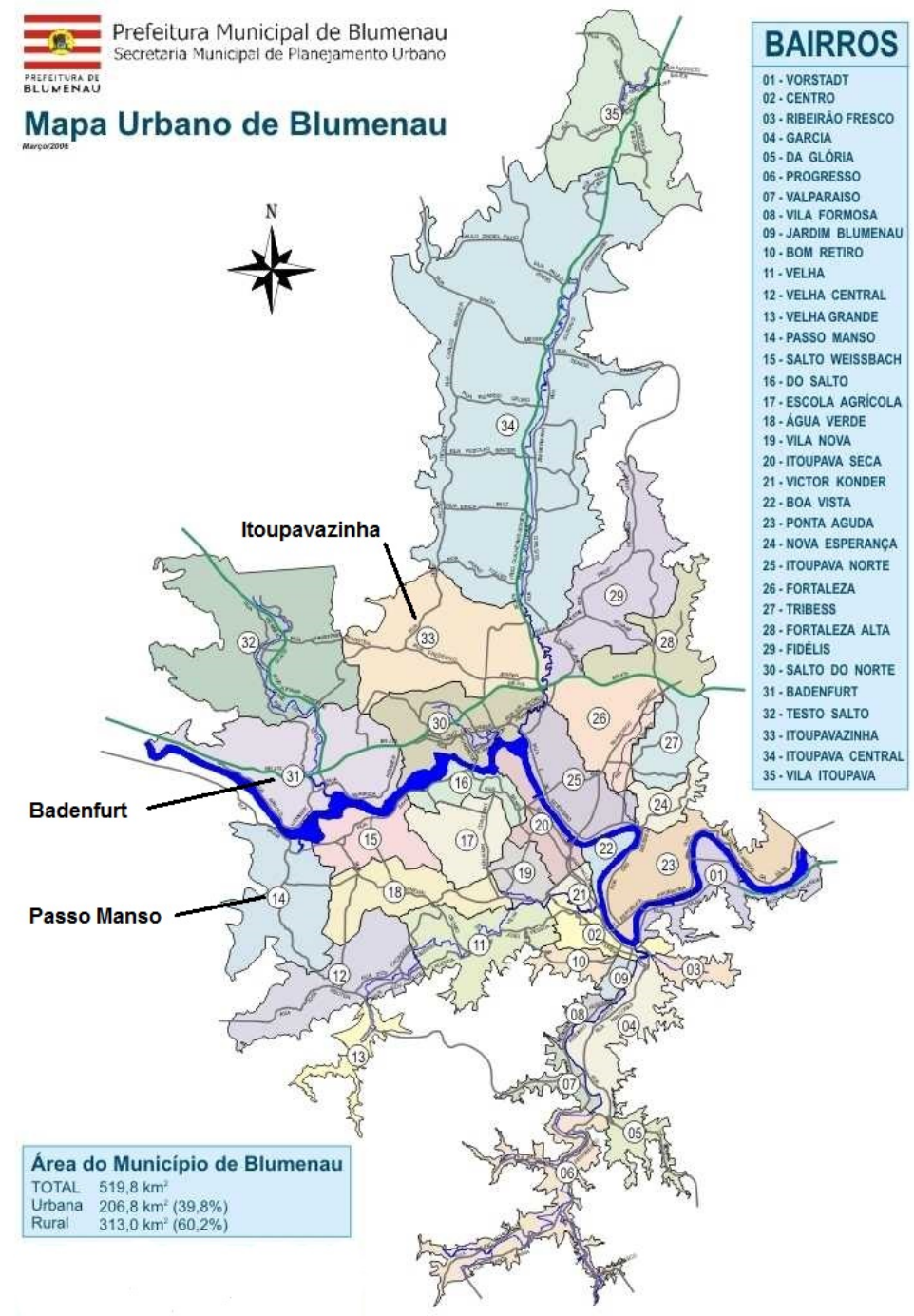

Figura 1: Localização dos três Conjuntos Habitacionais. Fonte: Prefeitura Municipal de Blumenau - PMB.

Adiante é apresentado na Figura 2 uma caracterização fotográfica dos três Conjuntos Habitacionais avaliados: Morada das Figueiras, Hamburgo, e Parque da Lagoa.
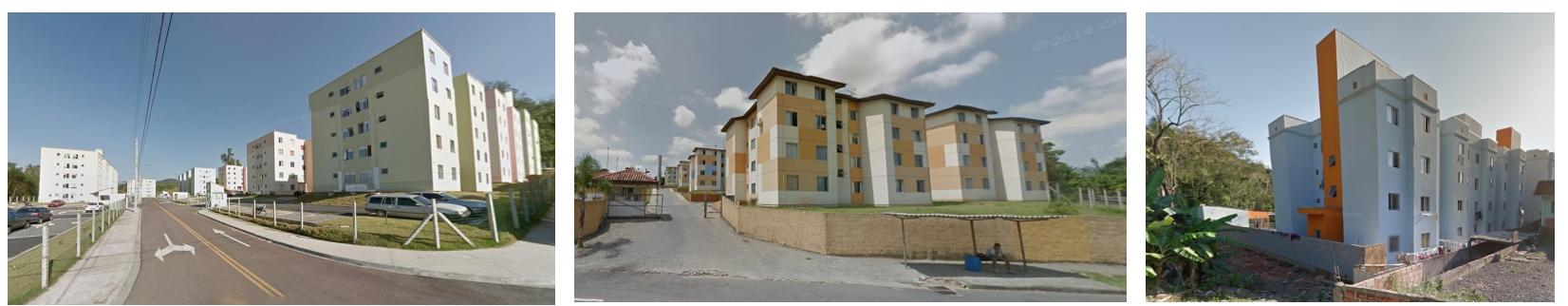

Figura 2 - Vista exterior dos Conjuntos Habitacionais

Os três Conjuntos Habitacionais foram concluídos respectivamente nas datas de novembro de 2010 - Residencial Parque da Lagoa; março de 2011 - Residencial Hamburgo; junho de 2011 - Residencial Morada das Figueiras. 
Na tabela 2 adiante são apresentados os resultados das análises realizadas.

Tabela 2: Resultados da avaliação realizada nos três Conjuntos Habitacionais analisados

\begin{tabular}{|c|c|c|c|c|c|c|c|c|c|}
\hline \multirow{2}{*}{$\begin{array}{l}\text { Situações de avaliação: } \\
A=\text { adequado; } P=\text { parcial; } I=\text { inadequado } \\
\text { Avaliações realizadas }\end{array}$} & \multicolumn{9}{|c|}{ Conjuntos Habitacionais } \\
\hline & \multicolumn{3}{|c|}{ Hamburgo } & \multicolumn{3}{|c|}{$\begin{array}{l}\text { Morada das } \\
\text { Figueiras }\end{array}$} & \multicolumn{3}{|c|}{$\begin{array}{l}\text { Parque da } \\
\text { Lagoa }\end{array}$} \\
\hline \multicolumn{10}{|l|}{ CONFORTO AMBIENTAL } \\
\hline Análise de projeto arquitetônico: & A & $\mathbf{P}$ & 1 & A & $\mathbf{P}$ & I & A & $\mathbf{P}$ & $\mathbf{I}$ \\
\hline Controle solar nas aberturas \& luz natural controlada & & & $\mathrm{x}$ & & & $\mathrm{x}$ & & & $\mathrm{x}$ \\
\hline Iluminação natural nos BWCs & $\mathrm{x}$ & & & $\mathrm{x}$ & & & $\mathrm{X}$ & & \\
\hline Ventilação natural cruzada & $\mathrm{x}$ & & & $\mathrm{x}$ & & & $\mathrm{X}$ & & \\
\hline Tratamento paisagístico com sombra arbórea no térreo & & & $\mathrm{x}$ & & & $\mathrm{x}$ & & & $\mathrm{x}$ \\
\hline \multicolumn{10}{|l|}{ Desempenho térmico: } \\
\hline$\rightarrow$ Paredes externas & $\mathrm{x}$ & & & $\mathrm{x}$ & & & $\mathrm{X}$ & & \\
\hline$\rightarrow$ Coberturas & & & $\mathrm{x}$ & & & $\mathrm{x}$ & $\mathrm{x}$ & & \\
\hline $\begin{array}{l}\text { Satisfação dos usuários quanto ao conforto ambiental: } \\
\text { entrevistas }\end{array}$ & & & & & & & & & $\mathrm{x}$ \\
\hline Excesso de ruído entre os vizinhos & & $\mathrm{x}$ & & & & $\mathrm{x}$ & & & $\mathrm{x}$ \\
\hline FUNCIONALIDADE & A & $\mathbf{P}$ & 1 & A & $\mathbf{P}$ & $\mathbf{I}$ & A & $\mathbf{P}$ & I \\
\hline Dimensão dos ambientes & & & $\mathrm{x}$ & & & $\mathrm{x}$ & & & $\mathrm{x}$ \\
\hline Satisfação dos usuários quanto à funcionalidade: entrevistas & & & $\mathrm{x}$ & & & $\mathrm{x}$ & & & $\mathrm{x}$ \\
\hline Dimensionamento da área de serviço e cozinha & & & $\mathrm{x}$ & & & $\mathrm{x}$ & & & $\mathrm{x}$ \\
\hline Falta espaço para estudo ou trabalho & & & $\mathrm{x}$ & & & $\mathrm{x}$ & & & $\mathrm{x}$ \\
\hline AMBIÊNCIA URBANA & A & $\mathbf{P}$ & 1 & A & $\mathbf{P}$ & $\mathbf{I}$ & A & $\mathbf{P}$ & $\mathbf{I}$ \\
\hline Equipamentos de apoio & $\mathrm{x}$ & & & & & & & & \\
\hline Escola & $\mathrm{x}$ & & & $\mathrm{x}$ & & & $\mathrm{x}$ & & \\
\hline Creche & & & $\mathrm{x}$ & $\mathrm{x}$ & & & $\mathrm{x}$ & & \\
\hline Posto de saúde ou Ambulatório & & $\mathrm{x}$ & $\mathrm{x}$ & $\mathrm{x}$ & & & & $\mathrm{x}$ & \\
\hline Comércio/serviços de apoio & & $\mathrm{x}$ & & & $\mathrm{x}$ & & & $\mathrm{x}$ & \\
\hline Parques/praças/equipamentos de lazer & & & $\mathrm{x}$ & & & $\mathrm{x}$ & $\mathrm{x}$ & & \\
\hline Transporte público & & & $\mathrm{x}$ & $\mathrm{x}$ & & $\mathrm{x}$ & $\mathrm{x}$ & & \\
\hline \multicolumn{10}{|l|}{ Áreas de convivência } \\
\hline \multicolumn{10}{|l|}{ Concentração e tratamento de espaços abertos: } \\
\hline $\begin{array}{l}\rightarrow \text { diferenciação entre espaços abertos e estacionamentos, } \\
\text { definindo funcionalmente os espaços; }\end{array}$ & & $\mathrm{x}$ & & & & $\mathrm{x}$ & & & $\mathrm{x}$ \\
\hline $\begin{array}{l}\rightarrow \text { demarcação de limites de segurança; privacidade e } \\
\text { identidade e o conforto no uso destes lugares; }\end{array}$ & & & $\mathrm{x}$ & & & $\mathrm{x}$ & & $\mathrm{x}$ & \\
\hline \multicolumn{10}{|l|}{ Segregação e tratamento de acessos: } \\
\hline$\rightarrow$ acessibilidade e segurança dos acessos desde a rua; & & & $\mathrm{x}$ & & & $\mathrm{x}$ & & & $\mathrm{x}$ \\
\hline $\begin{array}{l}\rightarrow \text { diferenciação dos percursos destinados aos pedestres e ao } \\
\text { trânsito de veículos; }\end{array}$ & & & $\mathrm{x}$ & & & $\mathrm{x}$ & & & $\mathrm{x}$ \\
\hline \multicolumn{10}{|l|}{ Características das vias circundantes aos edifícios: } \\
\hline $\begin{array}{l}\rightarrow \text { necessidade de segurança e boa ambiência, apresentando } \\
\text { conforto acústico e higiene; }\end{array}$ & & & $\mathrm{x}$ & & & $\mathrm{x}$ & & & $\mathrm{x}$ \\
\hline \multicolumn{10}{|l|}{ Racionalização da implantação dos estacionamentos: } \\
\hline $\begin{array}{l}\rightarrow \text { proporcionar redução do fluxo veicular, da área } \\
\text { pavimentada e sem ocupar áreas verdes; }\end{array}$ & & & $\mathrm{x}$ & & & $\mathrm{x}$ & & & $\mathrm{x}$ \\
\hline \multicolumn{10}{|l|}{ Localização das lixeiras coletivas: } \\
\hline $\begin{array}{l}\rightarrow \text { necessidade de salubridade, em lugar fechado e longe de } \\
\text { espaços de uso público; }\end{array}$ & & $\mathrm{x}$ & & & $\mathrm{x}$ & & & $\mathrm{x}$ & \\
\hline \multicolumn{10}{|l|}{ Cobertura na entrada dos edifícios: } \\
\hline $\begin{array}{l}\rightarrow \text { necessidade de proteção contra intempéries, } \\
\text { proporcionando demarcação de acesso principal; }\end{array}$ & & & $\mathrm{x}$ & & $\mathrm{x}$ & $\mathrm{x}$ & & & $\mathrm{x}$ \\
\hline
\end{tabular}


Continuação

\begin{tabular}{|c|c|c|c|c|c|c|c|c|c|}
\hline \multicolumn{10}{|l|}{ Adequação ao terreno natural: } \\
\hline $\begin{array}{l}\rightarrow \text { implantação da edificação com aproveitamento da } \\
\text { topografia natural, desníveis e cobertura do terreno; }\end{array}$ & & & $\mathrm{x}$ & & & $\mathrm{x}$ & & $\mathrm{x}$ & \\
\hline $\begin{array}{l}\rightarrow \text { nível de implantação dos apartamentos térreos para a } \\
\text { necessidade de privacidade e ambiência dos cômodos; }\end{array}$ & & & $\mathrm{x}$ & & & $\mathrm{x}$ & & & $\mathrm{x}$ \\
\hline \multicolumn{10}{|l|}{ Forma e tratamento externo dos edifícios: } \\
\hline $\begin{array}{l}\rightarrow \text { definição da morfologia do edifício para aproveitar os } \\
\text { fatores climáticos favoráveis, como o vento e a vegetação; }\end{array}$ & & $\mathrm{x}$ & & & & $\mathrm{x}$ & & & $\mathrm{x}$ \\
\hline $\begin{array}{l}\rightarrow \text { criação favorável ou não de microclimas; necessidade de } \\
\text { orientação e identidade. }\end{array}$ & & & $\mathrm{x}$ & & & $\mathrm{x}$ & & & $\mathrm{X}$ \\
\hline SUSTENTABILIDADE & A & $\mathbf{P}$ & $\mathrm{I}$ & A & $\mathbf{P}$ & $\mathrm{I}$ & A & $\mathbf{P}$ & $\mathbf{I}$ \\
\hline \multicolumn{10}{|l|}{ Sustentabilidade ambiental: } \\
\hline \multicolumn{10}{|l|}{$\rightarrow$ Proteção ecológica e agricultura urbana } \\
\hline Cumprimento da legislação ambiental & & $\mathrm{x}$ & & & $\mathrm{x}$ & & & & $\mathrm{x}$ \\
\hline $\begin{array}{l}\text { Compatibilização dos zoneamentos (urbano, ambiental e } \\
\text { balanço hídrico) }\end{array}$ & $\mathrm{x}$ & & & & & $\mathrm{x}$ & $\mathrm{x}$ & & \\
\hline $\begin{array}{l}\text { Provimento de áreas para agricultura urbana e paisagismo } \\
\text { produtivo }\end{array}$ & & & $\mathrm{x}$ & & $\mathrm{x}$ & & & & $\mathrm{x}$ \\
\hline \multicolumn{10}{|l|}{$\rightarrow$ Infraestrutura verde: } \\
\hline Gestão da água & & & $\mathrm{X}$ & & & $\mathrm{x}$ & & & $\mathrm{x}$ \\
\hline Drenagem natural & & & $\mathrm{X}$ & & $\mathrm{X}$ & & & & $\mathrm{x}$ \\
\hline Tratamento de esgoto alternativo & & & $\mathrm{x}$ & & & $\mathrm{x}$ & & & $\mathrm{x}$ \\
\hline \multicolumn{10}{|l|}{$\rightarrow$ Redução, reutilização e reciclagem de resíduos } \\
\hline \multicolumn{10}{|l|}{ Sustentabilidade social: } \\
\hline Urbanidade & & & $\mathrm{x}$ & & & $\mathrm{x}$ & & & $\mathrm{x}$ \\
\hline Comunidade com sentido de vizinhança & & & $\mathrm{x}$ & & $\mathrm{x}$ & & & $\mathrm{x}$ & \\
\hline Mobilidade e transporte sustentável & & & $\mathrm{x}$ & & $\mathrm{x}$ & & & $\mathrm{x}$ & \\
\hline
\end{tabular}

\subsection{Análise dos resultados}

No que se refere ao conforto ambiental, nos três empreendimentos os resultados foram semelhantes, principalmente no quesito de orientação solar dos apartamentos, devido ao padrão da tipologia de plantas baixas (rebatidas) adotadas nos projetos; desta forma, existem apartamentos com boa orientação solar e outros não. Nos três Conjuntos Habitacionais analisados do Programa Minha Casa Minha Vida, não são utilizados os recursos arquitetônicos de controle solar nas aberturas (venezianas e simulares), causando prejuízos como: "molhar dentro do apartamento" (como declarado por alguns moradores), insatisfação e desconforto em relação a iluminação natural, temperatura do ar e ventilação natural dentro dos apartamentos.

Os espaços coletivos dos Conjuntos encontram-se inadequados em relação ao tratamento paisagístico e sombra de árvores, pois são raros os espaços com sombra ao redor dos edifícios. No que se refere ao desempenho térmico de paredes externas e coberturas, avaliado com base nas recomendações do Método de Mahoney aplicado para Blumenau, as paredes externas em alvenaria de blocos cerâmicos autoportantes (com espessura final de $\sim 14 \mathrm{~cm}$ ) encontram-se adequadas em relação à transmitância térmica $(\mathrm{U})$ e à capacidade térmica $(\mathrm{Ct})$. Porém a situação da cobertura das edificações é inadequada, consistindo de telha de fibrocimento de $6 \mathrm{~mm}+$ câmara de ar + laje maciça de concreto armado com $10 \mathrm{~cm}$ de espessura. 
A avaliação dos moradores apontou para aberturas pequenas, com janelas do tipo de correr, com abertura de apenas metade do vão. Em dois dos três Conjuntos foi documentado que mais da metade das pessoas entrevistadas declarou haver excesso de ruídos internos, relatando ouvir a descarga do vizinho, a música, a televisão e quando em atividade na cozinha. Outro ponto foi à entrada de chuva pelas janelas, por não haver nenhum tipo de elemento de proteção externo (como venezianas e similares), inviabilizando o espaço próximo a janela ou prejudicando os materiais que estiverem expostos.

No que se refere a funcionalidade, em relação a dimensão dos ambientes (área mínima), as plantas baixas dos apartamentos se encontram na situação de inadequação nos três (3) Conjuntos avaliados, tem por base as áreas mínimas citadas na tabela 2 adiante, conforme apresentado por Palermo e Peixer (2013);

Tabela 3: Comparação de autores acerca da área mínima $\left(\mathrm{m}^{2}\right)$ das unidades habitacionais

\begin{tabular}{|l|c|c|c|}
\hline & Boueri (1989) & Silva (1982) & IPT (1988) \\
\hline Sala & 15,00 & 10,50 & $12,00-14,00$ \\
\hline Cozinha & 7,20 & 3,60 & $10,00-12,00$ \\
\hline Banheiro & 4,20 & 2,52 & $2,50-3,00$ \\
\hline Dormitório 1 & 14,00 & 7,75 & $9,00-11,00$ \\
\hline Dormitório 2 & 12,00 & 5,00 & $8,00-9,00$ \\
\hline Área serviço & 5,40 & 2,10 & --- \\
\hline Total & 57,80 & 31,47 & $43,00-52,00$ \\
\hline
\end{tabular}

Fonte: Adaptado de PALERMO, Carolina, PEIXER, Keila Tyciana. Programa Minha Casa Minha Vida: perfil populacional de dois conjuntos habitacionais de Blumenau - SC e o rebatimento no espaço da moradia.

No que se refere a ambiência urbana, identificou-se uma maior dominância de situações de inadequação nos três Conjuntos avaliados, a exceção da existência de equipamentos de apoio (com adequação), para os Conjuntos Morada das Figueiras e Parque da Lagoa.

No que se refere a sustentabilidade, identificou-se uma maior dominância de situações de inadequação em dois Conjuntos avaliados, a exceção do Morada das Figueiras, com avaliações parciais em vários critérios de análise.

\section{CONCLUSÕES}

A partir dos resultados analisados, foram classificados (em ordem de maior qualidade para de pior qualidade) os seguintes Conjuntos: Hamburgo, Morada das Figueiras e Parque da Lagoa.

O critério de inserção urbana teve pouca relevância nos resultados, sendo que em todos os conjuntos foi verificado como deficiente. É uma característica geral dos empreendimentos de interesse social estarem marginalizados espacialmente, com acesso precário a infraestrutura urbana básica, como postos de saúde, comércio e serviços (bancos, lotéricas, supermercados, farmácias, etc), equipamentos de lazer (praças, academias, parques) e transporte público.

Os espaços externos tiveram grande relevância, visto que são os locais onde as pessoas se expressam, interagem e devem conviver de forma harmoniosa, respeitando o espaço do outro. 
Os conjuntos não oferecem estruturas adequadas, como espaços para festas e reuniões, levando os moradores a invadirem os espaços alheios, não há espaços adequados para a integração entre as gerações, como hortas comunitárias, pomares, educação ambiental, aulas de artes e artesanatos.

Outro ponto relevante foi em relação à sensação de insegurança no conjunto. Muitas famílias não deixam os filhos brincarem na rua porque têm medo dos traficantes que ocupam os espaços externos. As crianças ficam ociosas dentro de casa, pois não há atividades para fazerem, sendo que o pai e a mãe trabalham geralmente em período integral e não têm controle da rotina dos filhos, e as escolas não têm capacidade de permanência integral das crianças. Além disso, os conjuntos estão em bairros isolados, em ruas com pouca vitalidade e grandes vazios urbanos. Os portões de entrada estão sempre abertos e as rondas policiais são ineficientes. Nos três conjuntos houve relatos de violência e mortes, em períodos diversos do dia.

\section{AGRADECIMENTOS}

Os autores agradecem ao Povo do Estado de Santa Catarina, por meio dos recursos financeiros da Bolsa de Pesquisa FUMDES Art. 171 anos 2015-2016, que financiaram o apoio no desenvolvimento da pesquisa aqui relatada.

\section{REFERÊNCIAS}

Amore, C. S.; Shimbo, L. Z.; Rufino, M. B. (Orgs.). (2015). Avaliação do Programa Minha Casa Minha Vida em seis estados brasileiros. Rio de Janeiro: Letra Capital.

Carmo Filho, Jairson Jairo do; Veloso, Maísa Fernandes Dutra (2005). Avaliação de pós-ocupação de residências em Natal/RN, a partir das recomendações de Armando de Holanda para a construção no Nordeste. In Anais... ENCAC-ELACAC 2005 Encontro Nacional e Encontro Latino-Americano de Conforto no Ambiente Construído. Maceió, Alagoas.

Coelho-De-Souza, Carolina Herrmann; Reimann, Daniel Eduardo; Fioretti, Giovana Bernini; Moehlecke, Juliana; Winckler, Márcia Meira; Sattler, Miguel Aloysio; Baptista, Silvia (2009). Percepção de conforto ambiental na habitação popular Casa alvorada: terceira avaliação pós-ocupação. In Anais... X Encontro Nacional e VI Latino Americano de Conforto no Ambiente Construído. Natal RN.

Instituto Brasileiro de Geografia e Estatística - IBGE (2016). Estimativas da população residente nos municípios e para as unidades da federação brasileiros com data de referência em 10 de julho de 2016: Rio de Janeiro.

Kowaltowski, D. C. C. K., Granja, A. D., Mikami, S. A., Pina, G., \& Barros, L. A. F. (2009). Os Conceitos de Satisfação e Valor Desejado na Avaliação Pós-Ocupação em Habitação Social. In Anais... X Encontro Nacional e VI Encontro Latino-Americano de Conforto no Ambiente Construído, 1516-1525.

Mendes, Ana Luisa C. M.; Silveira, Ana Lúcia R. C. da (2010). Avaliação pós-ocupação em conjunto habitacional multifamiliar com ênfase no Conforto ambiental em Teresina-PI. In Anais... NUTAU 2010 - DESIGN E INOVAÇÃO: MENSAGENS E PRODUTOS PARA AMBIENTES SUSTENTÁVEIS. São Paulo, SP. 
Ornstein, S. W. (2017). Avaliação Pós-Ocupação (Apo) no Brasil, 30 Anos: O Que há de Novo?. Revista Projetar-Projeto e Percepção do Ambiente, 2(2), 7-12.

Ornstein, S., Ono, R., \& Oliveira, F. (2017). Em busca da qualidade na habitação social no Brasil: instrumentos para a Avaliação Pós-Ocupação (APO) aplicada a sistemas construtivos inovadores. In Anais... 4 CONGRESSO INTERNACIONAL DA HABITAÇÃO NO ESPAÇO LUSÓFONO. Covilhã, Universidade Beira Interior (pp. 49-60).

Ornstein, Scheila; Romero, Marcelo (1992). Avaliação pós-ocupação (APO) do ambiente construído. São Paulo: Editora da Universidade de São Paulo.

Palermo, Carolina, Peixer, Keila Tyciana (2013). Programa Minha Casa Minha Vida: perfil populacional de dois conjuntos habitacionais de Blumenau - SC e o rebatimento no espaço da moradia. In Anais... 6o PROJETAR - O Projeto como Instrumento para a Materialização da Arquitetura: ensino, pesquisa e prática. Salvador.

Prefeitura Municipal de Blumenau - PMB (2006). Mapa Urbano de Blumenau. Secretaria de Planejamento - SEPLAN.

Rodrigues, Helena da Silva; Castro, Jorge Azevedo de; Rheingantz, Paulo Afonso; Santos, Isabelle Soares (2006). Matriz de descobertas: uma ferramenta para Avaliação pós-ocupação. In: ENTAC 2006 A Construção do Futuro. In Anais... XI Encontro Nacional de Tecnologia do Ambiente Construído. Florianópolis SC.

Vergara, Lizandra Garcia Lupi; Christmann, Laura Landerdahl ; Pires, Mariana Rohde; Noal, Roberta Bica (2007). Avaliação pós-ocupação das funções estar, serviço e dormir em ambientes residenciais de Santa Maria RS. In Anais... IX Encontro Nacional e V Latino Americano de Conforto no Ambiente Construído. Ouro Preto MG.

Villa, S. B.; Saramago, R. C.; Alves, C. A. (2015). Avaliação pós-ocupação em edifícios de apartamentos: a qualidade espacial e ambiental em Ribeirão Preto/SP. In Anais... SIMPÓSIO BRASILEIRO DE QUALIDADE DO PROJETO NO AMBIENTE CONSTRUÍDO, SBQP 4., 2015, VIÇOSA-MG. ANAIS... VIÇOSA-MG: UFV.

Villa, S. B.; Lemos, S. M.; Salustiano, L. R.; Ribeiro, G. P. N. (2016). Inovação tecnológica na avaliação pós-ocupação: ferramentas digitais e interativas. In: ENCONTRO NACIONAL DE TECNOLOGIA DO AMBIENTE CONSTRUÍDO, 2016, São Paulo. In Anais... XVI Encontro Nacional de Tecnologia do Ambiente Construído: desafios e perspectivas da internacionalização da construção (ENTAC 2016). São Paulo: ANTAC, 2016. v. 1. p. 34393452. 